\begin{tabular}{|c|c|c|c|c|}
\hline \multicolumn{4}{|c|}{ НАУЧНО-ТЕХНИЧЕСКИЙ ВЕСТНИК ИНФОРМАЦИОННЫХ ТЕХНОЛОГИЙ, МЕХАНИКИ И ОПТИКИ } & \multirow[b]{2}{*}{ 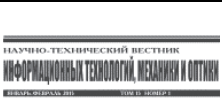 } \\
\hline : & $\begin{array}{cc}\text { март-апрель } 2016 & \text { Tом } 16 \text { № } 2 \\
\text { SCIENTIFIC AND TECHNICAL JOURNAL O }\end{array}$ & $\begin{array}{l}\text { ISSN 2226-1494 } \\
\text { NFORMATION TEC }\end{array}$ & $\begin{array}{l}\text { http://ntv.ifmo.ru/ } \\
\text { IECHANICS AND OPTICS }\end{array}$ & \\
\hline УНИВЕРСИТЕТ ИТМО & March-April 2016 & ISSN 2226-1494 & http://ntv.ifmo.ru/en & \\
\hline
\end{tabular}

\author{
УДК 53.089.68 \\ СОВЕРШЕНСТВОВАНИЕ ЭТАЛОННОЙ БАЗЫ В ОБЛАСТИ \\ МЕТРОЛОГИЧЕСКОГО ОБЕСПЕЧЕНИЯ РЕЗЬБОВЫХ СОЕДИНЕНИЙ \\ Ю.Г. Захаренко ${ }^{\text {, Н.А. Кононова }}{ }^{\text {a }, ~ А . А . ~ М о с к а л е в ~}{ }^{a}$ Э.Ф. Аскарова ${ }^{a}$, Е.А. Михеев \\ ${ }^{\mathrm{a}}$ ФГУП «ВНИИМ им. Д.И. Менделеева», Санкт-Петербург, 190005, Российская Федерация \\ b ОП «УЭ КЗС» ОАО «Метрострой», Санкт-Петербург, 190013, Российская Федерация \\ Адрес для переписки: A.A.Mosckalev@vniim.ru \\ Информация о статье \\ Поступила в редакцию 25.11.15, принята к печати 19.01.16 \\ doi: 10.17586/2226-1494-2016-16-2-338-344 \\ Язык статьи - русский \\ Ссылка для цитирования: Захаренко Ю.Г., Кононова Н.А., Москалев А.А., Аскарова Э.Ф., Михеев Е.А. Совершенствование \\ эталонной базы в области метрологического обеспечения резьбовых соединений // Научно-технический вестник информационных \\ технологий, механики и оптики. 2016. Т. 16. № 2. С. 338-344. doi: 10.17586/2226-1494-2016-16-2-338-344
}

\title{
Аннотация
}

Рассмотрены основные законодательные и прикладные проблемы метрологического обеспечения резьбовых соединений в Российской Федерации и за рубежом. Изложены основные недостатки существующих методов и средств измерения основного параметра резьбовых калибров - среднего диаметра резьбы. Представлено описание государственного рабочего эталона 1 разряда единицы длины - метра, введенного в эксплуатацию во Всероссийском научноисследовательском институте метрологии им. Д.И. Менделеева, с указанием основной области применения и метрологических характеристик эталона в соответствии с действующей государственной поверочной схемой. Показаны возможности применения эталона в области измерений параметров резьбовых калибров. Приведены технические решения, направленные на снижение составляющих неопределенности измерений среднего диаметра резьбы с использованием эталона: обеспечение стабильных температурных условий при эксплуатации эталона, снижение влияния внешних вибраций на процесс измерения, разработка специализированного программного обеспечения, использование которого снижает человеческий фактор и повышает степень автоматизации при осуществлении процесса измерений. Предложенные решения позволили достичь величины расширенной неопределенности измерений среднего диаметра резьбовых калибров 0,7-1,0 мкм. Сформулированы перспективные с точки зрения авторов направления деятельности по дальнейшему развитию и совершенствованию системы метрологического обеспечения резьбовых соединений.

Ключевые слова

резьбовые соединения, резьбовые калибры, средний диаметр, государственный эталон

\section{IMPROVEMENT OF REFERENCE BASE IN THE FIELD OF METROLOGICAL ASSURANCE OF THREAD JOINTS}

Yu.G. Zakharenko ${ }^{a}$, N.A. Kononova ${ }^{a}$, A.A. Mosckalev ${ }^{a}$, E.F. Askarova ${ }^{a}$, E.A. Mikheyev ${ }^{b}$ a D.I. Mendeleyev Institute for Metrology (VNIIM), Saint Petersburg, 190005, Russian Federation
b JSC “Metrostroy" Separate Subdivision “Applications Directorate of Protection Facilities Complex”, Saint Petersburg,
190013, Russian Federation

Corresponding author: A.A.Mosckalev@vniim.ru

Article info

Received 25.11.15, accepted 19.01.16

doi: 10.17586/2226-1494-2016-16-2-338-344

Article in Russian

For citation: Zakharenko Yu.G., Kononova N.A., Mosckalev A.A., Askarova E.F., Mikheyev E.A. Improvement of reference base in the field of metrological assurance of thread joints. Scientific and Technical Journal of Information Technologies, Mechanics and Optics, 2016, vol. 16, no. 2, pp. 338-344. doi: 10.17586/2226-1494-2016-16-2-338-344

\section{Abstract}

We consider the main and essential legislative and applicative issues of metrological assurance of thread joints in the Russian Federation and international practice. Basic limitations of the existing measuring methods of the main thread gage parameter - the pitch diameter of thread - are represented. We give the description of the first echelon state working calibration standard for linear unit, placed in service in D.I. Mendeleyev Institute for Metrology (VNIIM), including the general field of applications and metrological characteristics according to the state verification schedule. The possibilities of etalon application in the field of measurements of thread gages parameters are shown. Technical solutions for reducing the components of uncertainty of measurements of the pitch diameter using the etalon are described: temperature stability; reducing of external vibration effects on measuring procedure; developing of the special software for minimizing human 
factor and increasing automation level of the measuring procedure. The solutions described have enabled to achieve expanded uncertainty value about 0.7-1.0 microns. As a result, we also propose further ways for development and improvement of the system of metrology assurance in the field of thread joints.

Keywords

thread joints, thread gages, pitch diameter, national standard

\section{Введение}

Резьбовые соединения широко используются во многих отраслях промышленности и науки на протяжении последних десятилетий. В зависимости от области применения к резьбовым соединениям предъявляются различные требования по надежности и износостойкости (например, в случае, когда эксплуатация соединения подразумевает большое количество циклов сборки и разборки, либо при работе в экстремальных условиях, в вакууме, при приложении сложной разнонаправленной нагрузки в узлах, работающих под давлением, и пр.). Интенсивный рост и развитие промышленности (главным образом отраслей нефте- и газодобывающего комплекса, аэрокосмической отрасли) ведут к повышению требований к надежности существующих и вновь выпускаемых резьбовых деталей. Поскольку эксплуатационные характеристики резьбового соединения во многом определяются точностью изготовления геометрических элементов резьбы $[1,2]$, возникает задача повышения точности контроля таких элементов, а следовательно, необходимо вести речь о метрологическом обеспечении в данной области.

Цель настоящей работы - исследование и совершенствование эталонной базы Всероссийского научно-исследовательского института метрологии им. Д.И. Менделеева (ВНИИМ) в области метрологического обеспечения резьбовых соединений.

\section{Актуальные проблемы метрологического обеспечения резьбовых соединений}

Формально все просто: для контроля резьбы на рабочих деталях применяют резьбовые калибры (рис. 1), по характеру свинчивания которых с рабочей резьбой определяют пригодность последней к применению (порядок такой процедуры описывается в нормативных документах на калибры). Резьбовые калибры, в свою очередь, подвергают поэлементному контролю с помощью различных средств измерений, более высоких по точности. Традиционно - это координатно-измерительные машины (КИМ) либо горизонтальные длиномеры.

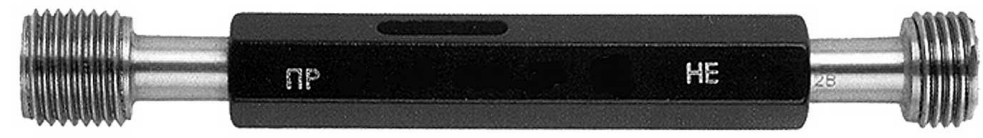

Рис. 1. Резьбовые калибры-пробки для контроля внутренней резьбы

На самом деле в метрологическом обеспечении резьбовых соединений есть ряд существенных проблем законодательного и прикладного характера.

Несмотря на кажущуюся простоту вопроса, в отечественной и зарубежной метрологии нет однозначного подхода к классификации резьбовых калибров как средств измерений. Как ни странно, но резьбовой калибр - мера, несущая информацию о целом ряде взаимосвязанных геометрических параметров разного типа, - формально не является средством измерения. А следовательно, нет поверочных схем, строго регламентирующих порядок передачи единицы длины от калибров к рабочим резьбам, нет единообразия во взгляде на пригодность тех или иных калибров для контроля резьб разной точности, нет четких требований к перечню параметров и способу их выражения, необходимых для наиболее полной и объективной оценки точности изготовления резьбового калибра. Ситуация усугубляется также ввиду того, что метрологические и технические требования к резьбовым калибрам отличаются в разных странах. Строгого соответствия между российскими и международными нормативными документами в этой области нет, а работы по гармонизации стандартов не получили широкого распространения. При этом многие российские промышленные предприятия из-за известных проблем с техническим оснащением на внутреннем рынке были вынуждены закупать резьбовые калибры за рубежом [3].

Кроме того, существует ряд предприятий, выпускающих специфические резьбовые соединения с узкой областью применения, обладающие характеристиками, не подлежащими объективному контролю с помощью резьбовых калибров, изготовленных по существующим стандартам. Такие предприятия вынуждены самостоятельно разрабатывать резьбовые калибры по собственным чертежам, при этом формальный статус таких калибров остается неопределенным.

Нельзя не упомянуть в данном контексте о Государственном реестре средств измерений Российской Федерации. Калибры для замковой резьбы производства московского ЗАО «РиСК» на сегодняшний день являются единственными калибрами утвержденного типа. Процедура внесения в реестр была осуществлена в начале 2000-х годов, и в данном случае замковые резьбы получили приоритет, очевидно, ввиду резкого повышения технико-экономического интереса к нефтедобывающей отрасли, наблюдавшегося в тот период времени (именно здесь находит основное применение данный тип резьбового соедине- 
ния). Такая ситуация влечет за собой следующую проблему - отсутствие метрологического обеспечения специализированных приборов для измерений параметров резьбы.

Не менее серьезные проблемы связаны с прикладными задачами измерений параметров резьбы и резьбовых калибров. В Российской Федерации функционируют более сотни региональных центров стандартизации и метрологии. Из них услуги по измерениям резьбовых калибров предлагают около трети [4]. При этом большинство лабораторий работают с ограниченной номенклатурой типов калибров в узком диапазоне.

Рассмотрим основные геометрические параметры резьбового соединения в сечении плоскостью, проходящей через ось резьбы (рис. 2).

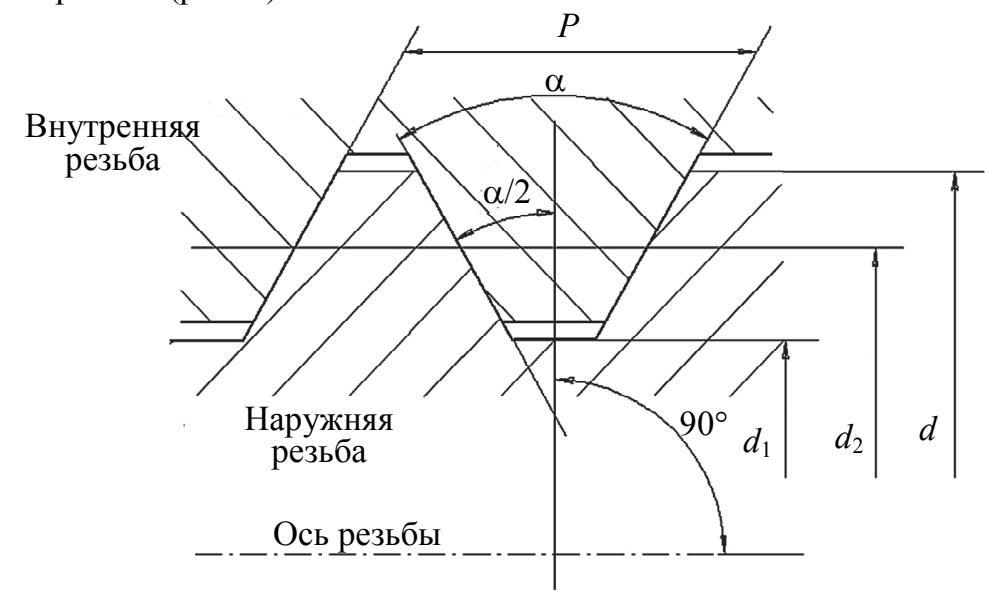

Рис. 2. Основные геометрические параметры резьбы

Согласно стандарту на термины и определения в области резьбы ГОСТ $11708-82^{1}$, основными параметрами цилиндрической резьбы являются следующие.

Наружный диаметр резьбы $(d)$ - диаметр цилиндра, описанного вокруг вершин наружной резьбы или впадин внутренней.

Внутренний диаметр резьбы $\left(d_{1}\right)$ - диаметр цилиндра, вписанного во впадины наружной резьбы или в вершины внутренней резьбы.

Средний диаметр резьбы $\left(d_{2}\right)$ - диаметр соосного с резьбой цилиндра, образующая которого пересекает профиль резьбы в точках, где ширина канавки равна половине шага резьбы.

Шаг резьбы $(P)$ - расстояние между соседними одноименными сторонами профиля в направлении, параллельном оси резьбы.

Угол профиля резьбы $(\alpha)$ - угол между боковыми сторонами профиля резьбы (для резьб с симметричным профилем нормируется величина $\alpha / 2$ - половина угла профиля).

Наиболее важным из приведенных выше параметров является средний диаметр [4, 5], для некоторых типов калибров его размер задается с погрешностью не выше \pm 3 мкм. Именно по среднему диаметру происходит сопряжение наружной и внутренней резьбы, однако диаметр этот соответствует цилиндру, построенному теоретически и проходящему частично в теле калибра, что, очевидно, усложняет процесс измерения. Фактически все методы измерений среднего диаметра являются косвенными, в чем и состоит их основной недостаток. Гораздо хуже ситуация обстоит с калибрами конической замковой резьбы: некоторые виды калибров изготавливаются так, что в основной плоскости (в которой задается средний диаметр) вообще отсутствует нарезанная резьба, т.е. величину диаметра здесь можно получить только расчетными методами и никак иначе $[6,7]$.

Таким образом, наиболее ответственный параметр калибра является в то же время наиболее сложным для измерения. В связи с этим, когда мы говорим об измерении резьбовых калибров, речь идет в первую очередь именно об измерении среднего диаметра.

Существует два метода измерений среднего диаметра: проекционный метод и метод трех проволочек. Основа проекционного метода - построение контура исследуемого резьбового калибра с помощью оптической проекционной системы и последующая его обработка. Данный метод технически реализуется, в частности, с использованием универсальных измерительных микроскопов (УИМ) и проекционных двухкоординатных измерительных приборов (ДИП).

Метод имеет два существенных недостатка. Во-первых, с помощью такого метода, очевидно, можно измерять только диаметры калибров-пробок. Получить изображение профиля резьбы калибра-кольца в

\footnotetext{
${ }^{1}$ ГОСТ 11708-82. Основные нормы взаимозаменяемости. Резьба. Термины и определения. Введен. 01.01.1984. М.: Изд-во стандартов, 1987. 33 с.
} 
проходящем свете в принципе невозможно. Во-вторых, метод имеет высокую погрешность ввиду того, что получаемое проекционное изображение профиля резьбы не является изображением осевого сечения.

Пределы допускаемой абсолютной погрешности измерения среднего диаметра резьбы проекционным методом составляют $\pm(2,5-4,5)$ мкм.

Метод трех проволочек для измерения среднего диаметра резьбы является более универсальным и, соответственно, более распространенным. Реализуется он с использованием горизонтальных длиномеров, оптиметров, реже с помощью КИМ.

Теория метода такова: во впадины резьбы, соответствующие одному витку, с противоположных сторон закладываются гладкие цилиндрические проволочки. Их диаметр рассчитывается из условия касания образующих проволочек с профилем резьбы в точках, принадлежащих среднему диаметру. Далее производят контактные измерения общего размера калибра с установленными проволочками, из которого величина среднего диаметра находится математически [8-11]. Аналогично измеряется и средний диаметр внутренней резьбы калибров-колец, но в этом случае цилиндрические проволочки заменяются на сферические наконечники щупа длиномера или КИМ.

Основным недостатком метода является то, что в общем случае при расчете среднего диаметра необходимо учитывать ряд поправок. Среди них, например, поправка на диаметры используемых проволочек, связанная с тем, что действительные значения диаметров могут отличаться от номинального. Пределы допускаемой абсолютной погрешности измерения среднего диаметра резьбового калибра методом трех проволочек с использованием горизонтального длиномера составляют, как правило, $\pm(1,5-2,5)$ мкм.

С целью выработки единого подхода к порядку применения калибров и передаче единицы длины от резьбовых калибров к рабочим резьбам во ВНИИМ с 2001 г. ведется работа по созданию системы метрологического обеспечения резьбовых калибров [12]. В 2014 г. во ВНИИМ утвержден государственный рабочий эталон 1 разряда единицы длины - метра в диапазоне $10^{-6}-1100$ мм, внедрение которого направлено, в том числе и на развитие и совершенствование эталонной базы в области измерений резьбовых калибров.

\section{Описание и основные характеристики эталона ВНИИМ}

B основе эталона положен горизонтальный длиномер Labconcept Nano швейцарской фирмы «TRIMOS» (рис. 3). Основной областью применения эталона являются высокоточные измерения эталонных плоскопараллельных концевых мер длины и эталонных измерительных колец. Изначально перед коллективом ВНИИМ стояла задача разработки государственного рабочего эталона единицы длины на базе горизонтального длиномера. Этим обусловлен выбор данной модели длиномера, а также ряд технических решений, направленных на создание соответствующих условий его содержания и эксплуатации.

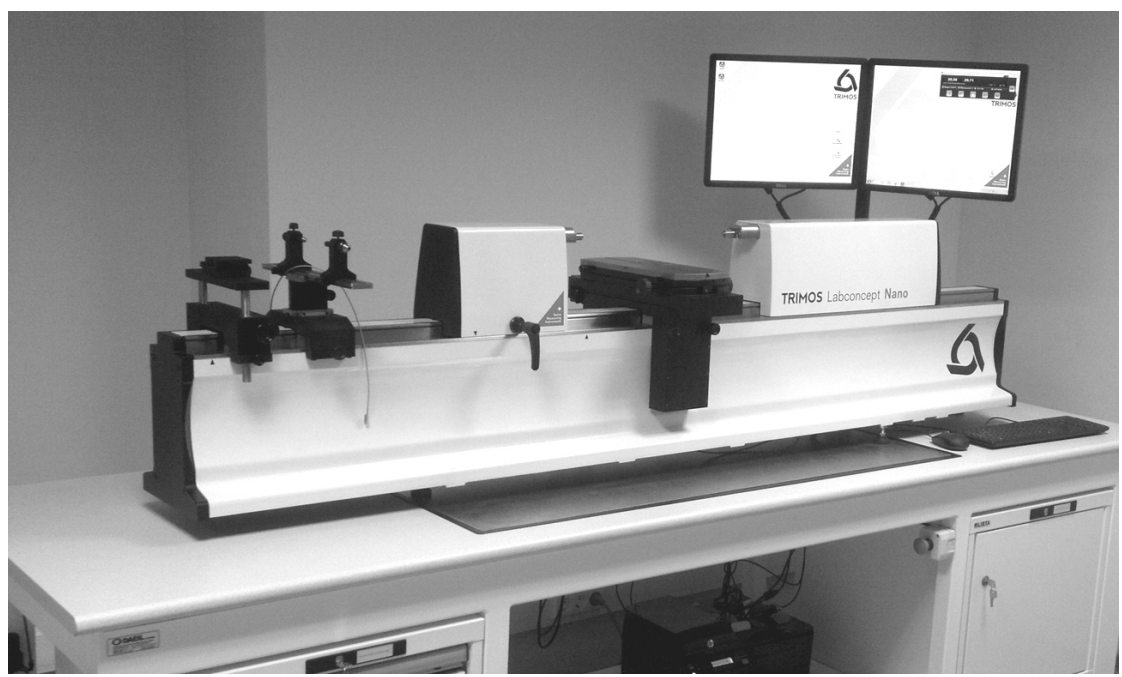

Рис. 3. Общий вид длиномера из состава эталона

Эталон установлен в отдельной, специально подготовленной лаборатории. Планировка позволила поместить длиномер и системы термостабилизации таким образом, чтобы создать в помещении стабильное температурное поле с равномерным распределением температуры по всему объему. В помещении круглосуточно поддерживается температура $(20,0 \pm 0,2){ }^{\circ} \mathrm{C}$. Кроме того, эталон снабжен системой температурной компенсации, в реальном времени учитывающей температуру длиномера и измеряемого объекта, с автоматическим внесением поправок в результат измерений.

Чтобы избежать влияния внешних вибраций на процесс измерений, длиномер установлен на специально спроектированном виброзащитном столе. Эталон также снабжен пневматической системой с 
обратной связью, автоматически регулирующей горизонтальное положение длиномера и компенсирующей изменение центра тяжести при перемещении подвижных кареток и измеряемого объекта, что важно при работе с резьбовыми калибрами-пробками, масса которых при больших номинальных диаметрах достигает десятков килограмм.

Пределы допускаемой абсолютной погрешности измерений согласно разряду по государственной поверочной схеме для средств измерений длины соответствуют $\pm(0,05+0,5 \cdot L)$ мкм, где $L-$ измеряемая величина в метрах. Эта характеристика улучшена на 30\% по сравнению с данными, заявленными изготовителем, и подтверждена результатами исследований с использованием государственного вторичного эталона единицы длины, а также эталонных плоскопараллельных концевых мер длины и эталонных измерительных колец [13].

\section{Особенности измерений резьбовых калибров на эталоне ВНИИМ}

При использовании специальных аксессуаров (цилиндрических проволочек, Т-образных щупов) на эталоне ВНИИМ можно выполнять измерения среднего диаметра резьбовых калибров (колец и пробок) по методу трех проволочек.

Помимо описанных выше технических решений, связанных с обеспечением требований к эталону, сам длиномер имеет ряд конструктивных особенностей, отличающих его от аналогов. Рассмотрим эти особенности в контексте процесса измерения среднего диаметра резьбы.

Перемещение измерительной каретки длиномера осуществляется только в автоматическом режиме с помощью соответствующих функций в программном обеспечении (ПО). Такое решение позволяет полностью исключить ошибки, связанные со сбоем счета при перемещении каретки, так как скорость перемещения ограничена и не может превышать значений, предусмотренных технической документацией.

Измерительный стол длиномера также снабжен электроприводом, управляющим вертикальным и горизонтальным перемещением. Величина перемещения задается через ПО с погрешностью не более \pm 1 мкм. Такое решение позволяет точно позиционировать калибр относительно измерительных проволочек или щупов. Это особенно важно при необходимости проведения измерений среднего диаметра калибра-пробки в нескольких сечениях на расстоянии, кратном шагу резьбы, а также в случае измерения калибров-колец для точного перемещения щупа вдоль оси резьбы при введении сферического наконечника в контакт с противоположными впадинами.

Оснащение измерительной каретки и стола длиномера управляемыми приводами обеспечивает автоматический поиск точек контакта щупа с поверхностью калибра, лежащих в плоскости, соответствующей диаметру. При измерении калибров-пробок достаточно грубого позиционирования оси калибра вблизи измерительной оси длиномера, поскольку используемые при этом измерительные проволочки входят в контакт с плоскопараллельными наконечниками длиномера по линии. При измерении калибровколец (не только резьбовых, но и в первую очередь гладких измерительных) контакт сферического наконечника щупа с поверхностью калибра осуществляется в точке, а следовательно, отклонение оси измерения от плоскости, соответствующей диаметральному сечению калибра, вносит погрешность в результат измерения. По сути, при ошибке позиционирования калибра мы получаем результат, в действительности не соответствующий измеряемому параметру.

Длиномер снабжен системой автоматического задания и приложения измерительного усилия с дискретностью 0,01 Н. Таким образом, исключается погрешность, связанная с разным характером приложения измерительного усилия при многократных измерениях среднего диаметра, а также обеспечивается симметричность приложения измерительного усилия при контакте щупа с противоположными сторонами профиля резьбы при измерении среднего диаметра калибров-колец.

Кроме того, авторами настоящей работы было разработано специализированное программное обеспечение «Thread Gages Calculation Software» (TGCS), предназначенное для автоматического вычисления величины среднего диаметра резьбового калибра по результатам косвенных измерений, полученных на эталоне. Область применения ПО распространяется на цилиндрические калибры-пробки и калибры-кольца метрической, трапецеидальной и трубной резьбы. Особенностью программы является полная совместимость с оригинальным комплексом ПО «WinDHI», управляющего измерениями с использованием эталона. Использование TGCS исключает человеческий фактор из процесса обработки результатов измерений, снижает трудоемкость при увеличении степени автоматизации измерений.

\section{Заключение}

Исследования государственного рабочего эталона 1 разряда единицы длины - метра с использованием резьбовых калибров-пробок и калибров-колец показывают, что совокупность описанных выше технических решений, направленных на совершенствование эталона, позволила обеспечить расширенную неопределенность измерений среднего диаметра резьбы на эталоне порядка $(0,7-1,0)$ мкм. Таким образом, метрологические характеристики в области измерений резьбовых калибров, полученные на эталоне 
ВНИИМ, не уступают лучшим аналогичным данным, заявленным ведущими зарубежными национальными метрологическими институтами $[14,15]$.

В качестве основных направлений деятельности по дальнейшему совершенствованию системы метрологического обеспечения резьбовых соединений можно предложить следующие:

- разработка новых высокоточных методов и средств измерений резьбовых калибров, в том числе на основе принципов лазерной интерферометрии;

- пересмотр и актуализация существующих нормативных документов в области резьбовых соединений;

- разработка и утверждение поверочных схем в области измерений геометрических параметров резьбовых соединений и резьбовых калибров с прослеживаемостью, в том числе, к эталону ВНИИМ;

- осуществление работ по внесению резьбовых калибров в Государственный реестр средств измерений Российской Федерации;

- совершенствование эталонных баз метрологических центров и институтов в области измерений резьбовых калибров;

- расширение международного сотрудничества в области метрологического обеспечения резьбовых соединений, организация и проведение сличений резьбовых калибров.

\section{Литература}

1. Лексиков В.П., Прокофьев А.Н. Износ витков резьбовых соединений в зависимости от метода обработки внутренней резьбы // Вестник Брянского государственного технического университета. 2006. № 2 (10). С. 83-84.

2. Москалев А.А. Особенности поверки калибров замковой резьбы // Материалы III Международного конкурса «Лучший молодой метролог КООМЕТ-2009». Минск, 2009. С. 123-128.

3. Шмаль Г.И., Кершенбаум В.Я., Хомяков А.И., Шнейдерман Я.А. и др. Семь раз отмерь... Круглый стол по метрологическим проблемам нефтегазового комплекса // Мир измерений. 2010. № 11. С. 7-15.

4. Захаренко Ю.Г., Кононова Н.А., Москалев А.А. Метрологическое обеспечение измерений. Резьбовые соединения // Контроль качества продукции. 2015. № 10. С. 41-45.

5. Merkac T.P., Acko B. Thread gauge calibration for industrial applications // Journal of Mechanical Engineering. 2010. V. 56. N 10. P. 637-643.

6. Сурков И.В., Суркова Е.А., Буртовая А.И., Мастрикова И.С. Разработка методики координатных измерений резьбовых конических калибров // Труды 25 научного симпозиума с международным участием "Метрология и метрологическое обеспечение". Созополь, Болгария, 2015. С. 478-486.

7. Буртовая А.И., Сурков И.В. Разработка математического обеспечения для размерно-точностного анализа результатов координатных измерений // В сб. Прогрессивные технологии в машиностроении. Челябинск: ЮУрГУ, 2012. С. 101-104.

8. Димов Ю.В. Метрология, стандартизация и сертификация. СПб.: Питер, 2013. 496 с.

9. Amin S.S., Raja J. Screw Thread Metrology. The University of North Carolina in Charlotte, USA, 2004.

10. Guidelines on the Determination of Pitch Diameter of Parallel Thread Gauges by Mechanical Probing. EURAMET cg-10. 2007. V. 1. 20 p.

11. NPL Notes on Screw Gauges [Электронный pecypc]. National Physical Laboratory, UK, 2010. 129 p. Peжим доступа: http://www.npl.co.uk/upload/pdf/screw_gauge_booklet.pdf, свободный (дата обращения 02.02.2016).

12.Поспелов Р.В., Позднышев В.Н., Абрамова Л.Ю., Баратов В.М., Хавинсон Л.Ф., Гершун М.А. Метрологическое обеспечение параметров замковых резьб и резьбовых соединений труб нефтяного сортамента // Надежность и сертификация оборудования для нефти и газа. 2002. № 1. С. 8-10.

13.Захаренко Ю.Г., Кононова Н.А., Москалев А.А., Фомкина З.В. Возможности «ВНИИМ им. Д.И. Менделеева» в области высокоточных измерений резьбовых соединений // Тезисы докладов Пятой всероссийской научно-техническая конференции Измерения и испытания в судостроении и смежных отраслях (Судометрика-2014). Санкт-Петербург, 2014. С. 148-152.

14. The BIPM key comparison database. Calibration and Measurement Capabilities. Length [Электронный ресурс]. 2013. Режим доступа: http://kcdb.bipm.org/appendixC/, свободный. Яз. англ. (дата обращения 30.09.2015).

15. Mosckalev A. High-accurate measurements of thread gages using the Labconcept NANO horizontal instrument // Proc. $24^{\text {th }}$ National Scientific Symposium with International Participation Metrology and Metrology Assurance. Sozopol, Bulgaria, 2014. P. 480-485.

Захаренко Юрий Гордеевич

Кононова Наталья Александровна кандидат технических наук, заместитель руководителя отдела, ФГУП «ВНИИМ им. Д.И. Менделеева», Санкт-Петербург, 190005, Российская Федерация, Y.G.Zackharenko@vniim.ru кандидат технических наук, руководитель отдела, ФГУП «ВНИИМ им. Д.И. Менделеева», Санкт-Петербург, 190005, Российская Федерация, N.A.Kononova@vniim.ru 
Москалев Андрей Андреевич

Аскарова Эльмира Флорисовна

Михеев Евгений Андреевич

Yury G. Zakharenko

Natalia A. Kononova

Andrey A. Mosckalev

Elmira F. Askarova

Evgeny A. Mikheyev инженер, ФГУП «ВНИИМ им. Д.И. Менделеева», Санкт-Петербург, 190005, Российская Федерация, A.A.Mosckalev@vniim.ru

- $\quad$ инженер, ФГУП «ВНИИМ им. Д.И. Менделеева», Санкт-Петербург, 190005, Российская Федерация, E.F.Askarova@vniim.ru

- $\quad$ руководитель отдела, ОП «УЭ КЗС» ОАО «Метрострой», СанктПетербург, 190013, Российская Федерация, miheev.ea@kzs-spb.ru

- $\quad \mathrm{PhD}$, Deputy head of the department, D.I. Mendeleyev Institute for Metrology (VNIIM), Saint Petersburg, 190005, Russian Federation, Y.G.Zackharenko@vniim.ru

$\mathrm{PhD}$, Head of the department, D.I. Mendeleyev Institute for Metrology (VNIIM), Saint Petersburg, 190005, Russian Federation, N.A.Kononova@vniim.ru

engineer, D.I. Mendeleyev Institute for Metrology (VNIIM), Saint Petersburg, 190005, Russian Federation, A.A.Mosckalev@vniim.ru

engineer, D.I. Mendeleyev Institute for Metrology (VNIIM), Saint Petersburg, 190005, Russian Federation, E.F.Askarova@vniim.ru

Head of the department, JSC "Metrostroy" Separate Subdivision "Applications Directorate of Protection Facilities Complex", Saint Petersburg, 190013, Russian Federation, miheev.ea@kzs-spb.ru 